\title{
Towards an economic evidence base for pharmacogenetics: consideration of outcomes is key
}

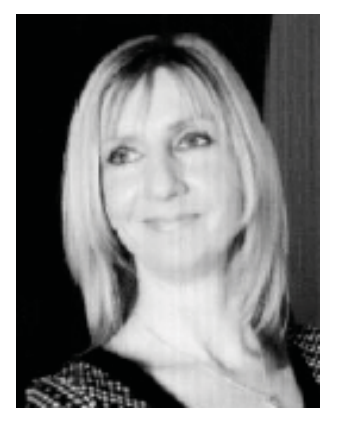

KatherinePayne,

${ }^{1} \mathrm{~T}$ he U niversity of

$M$ anchester, $\mathrm{H}$ ealth

Economics Research at

$M$ anchester (HERM AN),

$\mathrm{H}$ ealth M ethodology Research

Group, School of Community

Based M edicine, U niversity

Place, 0 xford Road,

$M$ anchester, M 13 9PL, U K

${ }^{2} \mathrm{~T}$ he $\mathrm{N}$ owgen Centre,

N owgen - A Centre for

$G$ enetics in $\mathrm{H}$ ealthcare,

29 Grafton Street,

$M$ anchester, M 13 9WU,

UK

Tel.: +44 1612768979 ;

Fax: +44 161276 4058;

E-mail: katherine.payne@

manchester.ac.uk

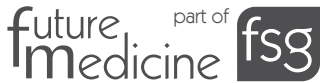

'In the absence of a robust a lternative, QALYs a re c urrently widely used by UK decision-makers trying to make diffic ult decisionsa bout how best to use healthcare resources for maximum patient benefit.'

Using genetic information to target the safe and effective use of medicines offers potential patient benefits but will also have an impact on the use of healthcare resources. Pharmacogenetics has been viewed as something for the future [1], but real clinical examples now exist $[2,3]$. Some pharmacogenetic tests, such as the thiopurine methyltransferase (TPM T) test that aims to predict the risk of severe neutropenia for the purine drugs azathioprine and 6-mercaptopurine, have relatively low unit costs of approximately $£ 28$ [101]. Even low unit cost tests may have a significant cost impact if they have a high volume of uptake in a healthcare system. In England, two clinical laboratories currently offer routine TPM T-enzyme level testing for purine drugs. In 2006, one laboratory reported analyzing 11,167 samples [101] and the second 10,931 samples [A M arinaki, Pers. Comm. 2007]. Assuming both laboratories charge $f 28$ [101] a test, the total cost of testing to the N H S is currently $£ 0.6$ million. As pharmacogenetic tests are provided from finite healthcare budgets, there is an opportunity cost associated with diverting funding towards them. Two important issues need to be considered. First, the cost of introducing a pharmacogenetic test includes more than just the cost of the test itself, and the impact on other healthcare resources, such as managing adverse drug reactions and frequency of blood monitoring, needs to be quantified. The types of cost to consider are driven by the perspective of the decision-maker who is responsible for how to spend a given budget. Second, there may be added value associated with introducing a pharmacogenetic test to guide a prescribing decision, in terms of improved health-related quality of life resulting from fewer severe side effects and improved treatment response in the patient population taking the medicine. This is a complex issue for decision-makers as they have to decide whether to divert scarce healthcare resources to pharmacogenetic testing. It is not clear that decision-makers currently have sufficient robust information on costs and patient benefits to allow an informed trade off.

Pharmacogenetic tests broadly fall into one of two types, those provided through clinical laboratories, such as the TPM T test, and those for which a product license has been granted in a similar way to new medicines, such as Third Wave Technologies' (WI, USA) Invader ${ }^{\circledR}$ U GT 1A1 M olecular Assay, which was approved by the US FDA in 2005. For all new medicines, a product licence must be granted before they can be prescribed. The licence will be awarded once the regulatory body is satisfied that there is sufficient information on the product's quality, safety and efficacy. In some countries, specific medicines are selected for further evaluation of evidence of clinical and cost-effectiveness. In England, this appraisal process is carried out by NICE. Laboratories providing genetics and pharmacogenetic tests are required to meet external quality assessment standards, but such tests do not go through the same licensing process as medicines and may emerge into clinical practice without supportive evidence on clinical utility or cost effectiveness.

'Dec ision makers need to considerwhich outcomes they deem most useful in the context of pharma cogenetic testing.'

Robust evidence on the economic impact of services and technologies is now considered by health departments and others to be a necessary prerequisite to inform clinical decision making, local and national policy. Economic evaluations potentially provide decision makers with such information and are formally defined as: 'the comparative analysis of alternative courses of action in terms of both their costs and consequences' [4]. It is clear, therefore, that the evaluation involves comparing a minimum of two interventions, one of which 
should be current practice, to allow the added value of the new technology to be appraised. The type of economic evaluation is defined by the approach taken to measure the consequences or outcomes in terms of the impact on the patient population receiving the technologies under comparison.

In the UK, NICE uses cost-effectiveness data and asks for outcome measures that are generic [5], rather than ones specific to particular conditions or alleviation of symptoms, to allow direct comparison of interventions with different effects. Examples of generic health status measures include the EQ -5D [6] and the SF-6D [7]. The outcome generally used by NICE is the qualityadjusted life year (QALY), which is the product of quality-of-life and survival [8]. Weightings (utilities) are assigned to the quality-of-life associated with being in a particular health state for each year of survival. Preference-based methods are used to calculate these utilities [5]. H ealthcare technologies are then prioritised in terms of those that maximise the improvement in QALYs for a given cost. The use of QALYs to prioritise resources is not without some problems and there have been a number of criticisms $[9,10]$.

\section{'...we need economic evaluations to inform the appropriate use of pharmacogenetic testing in clinic al practice.'}

O ne specific criticism of QALYs for geneticbased technologies is they may not capture all the potential benefits. Focussing on improved health status alone misses key benefits, such as the ability to make an informed life decision for the individual and family members [11]. Cost-benefit analysis uses a monetary valuation of outcome that allows consideration of such nonhealth and process attributes. Process attributes relate to benefits that accrue to patients as a result of how a service is provided, such as the value attached to who explains a particular pharmacogenetic test, and interprets the implications for prescribing [12]. Contingent valuation methods, such as willingness to pay (WTP) or the human capital approach, are used to attach a monetary value to the benefit. Collating WTP data involves asking a sample of respondents to value a hypothetical scenario describing the technology [13]. The relevance of WTP has been criticised in the U K where people are not used to paying for their healthcare, but the approach is used in countries with privately financed healthcare systems. Further criticisms of WTP center on the hypothetical nature of the exercise [14]. In the absence of a robust alternative, QALYs are currently used widely by UK decision-makers trying to make difficult decisions about how best to use healthcare resources for maximum patient benefit.

Pharmacogenetic tests will also compete for scarce healthcare resources and may also be subject to robust evaluation. Published economic evaluations of pharmacogenetic technologies are notable by their absence $[15,16]$ but the TPM T test is one example where there have been a number of published studies [17-22]. The primary aim of the TPM T test is to reduce the incidence of severe neutropenic episodes associated with purine drugs, and published economic evaluations have generally used this as the primary outcome measure. This is an intermediate rather than a final outcome because it does not tell a decision-maker the impact on overall benefit for a patient population who are tested. Five of the six published evaluations used a measure of 'neutropenia avoided' as the prime outcome of interest [17-19,21,22]. Dubinsky et al. used time to an initial or sustained response to azathioprine in people with Crohn's disease [20]. Frequency of leucopenic events was also estimated but there was no real observable difference in this outcome. $M$ arra et al. estimated a number needed to treat of 20 to avoid one adverse event over 6 months [17]. $\mathrm{O}$ h et al. assumed the probability of not stopping treatment in the first year due to serious adverse events was $99.9 \%$ for genotypebased dosing compared with $97.06 \%$ for conventional dosing of azathioprine [19]. Two of the models reported cost-per-life years gained by making assumptions, which were not based on data, about the number of deaths averted by introducing TPM T testing [18,22]. Winter et al. assumed that one death would be avoided by screening a population of 1000 people with inflammatory bowel disease [18]. Van den Akkervan $M$ arle et al. applied the same value and estimated that one death per 1000 children with acute lymphoblastic leukemia would be avoided by TPM T genotyping [22]. Priest et al. was the only model to include an estimate of the impact on QALYS [21]. Clinical opinion rather than data from patients was used to describe the EQ -5D health states associated with neutropenia and $\mathrm{N}$ ew Zealand social tariffs were used to estimate weightings for each of the health states [21]. This analysis assumed that no deaths would be avoided from TPM T testing. The enzyme level 
(phenotype) test was superior and avoided an estimated 71 cases of neutropenia compared with 40 neutropenias avoided by a genotype test. Small improvements in QALYs were assumed using a phenotype test or genotype test compared with no testing.

All of the published economic evaluations reported TPMT testing to be a cost-effective use of healthcare resources, but it is important to note these were retrospective modeling studies that used existing data to populate a model. Models represent simplifications of clinical pathways involved in the choice of whether or not to use a technology, such as pretreatment TPM T testing. N one of the studies identified any actual data that measured the impact of the TMPT test on the number of deaths averted (improved survival) or health-related qualityof-life. The analysts who populated these models therefore had to make assumptions and extrapolate from the assumed number of cases of neutropenia avoided by using the test to estimate the impact on life-years gained. D ubinsky et al. discussed how a calculation of QALYS gained was not possible due to lack of data [20]. Priest et al. described how they chose to use health-related quality-of-life estimates from clinical experts rather than patients because of convenience [21].

Commentators agree that we need economic evaluations to inform the appropriate use of pharmacogenetic testing in clinical practice
$[15,23,24]$. There is clearly also a need to consider which outcomes are to be used in such economic evaluations. The QALY has currently been labeled in the UK as the ideal outcome since it allows comparison between interventions used for different conditions and due to the absence of a more robust alternative measure to use. Decision makers need to consider which outcomes they deem most useful in the context of pharmacogenetic testing and whether it is sufficient to know the number of side-effects avoided by introducing the test. $O$ ne could argue that this information alone is not sufficient to divert healthcare resources to a pharmacogenetic technology and robust information that shows the link between averting side effects and improving the quantity and quality of life years compared with current clinical practice is also necessary. The question that remains unanswered is who will fund the research that is necessary to provide this information.

Financial \& competing interests disclosure

The author has no relevant affiliations or financial involvement with any organization or entity with a financial interest in or financial conflict with the subject matter or materials discussed in the manuscript. This includes employment, consultancies, honoraria, stock ownership or options, expert testimony, grants or patents received or pending or royalties.

$\mathrm{No}$ writing assistance was utilized in the production of this manuscript.

\section{Bibliography}

Papers of special note have been highlighted as either of interest $(\bullet)$ or of considerableinterest $(\cdot \bullet)$ to readers.

1. The Royal Society: Personalised medicines: H opes and Realities. London, UK (2005).

2. Fargher EA, Tricker $K, N$ ewman $B$ et al.: Current use of pharmacogenetic testing: a national survey of thiopurine methyltransferase (TPM T) testing prior to azathioprine prescription. J. Clin. Pharm. Ther. 32, 187-195 (2007).

3. Grabinski JL: Pharmacogenomics of anticancer agents: implications for clinical pharmacy practice. J. Pharm. Prac. 20(3), 246-251 (2007).

4. D rummond $M$, Sculpher $M$, Torrance G W, O 'Brien BJ, Stoddart G : $M$ ethods For the Economic Evaluation of $\mathrm{H}$ ealth $\mathrm{C}$ are Programmes. 3rd Revised Edition. O xford U niversity Press, O xford, UK (2005).
- Methods textbook for people with some experience of the design and conduct of economic evaluations.

5. N ational Institute for $\mathrm{H}$ ealth and $\mathrm{Clinical}$ Excellence: a Guide to Technology Appraisals. NICE, London, UK (2004).

6. D olan $\mathrm{P}: \mathrm{M}$ odelling valuation for EuroQ $\mathrm{OL}$ health states. M ed. Care 35, 351-363 (1997).

7. Brazier J, Roberts J, D everill $M$ : The estimation of a preference-based measure of health from the SF-36. J. Health Econ. 21(2), 271-292 (2002).

8. Williams A: Economics of coronary artery bypass grafting. BM J 291, 326-329 (1985).

- Introduced the concept of using quality-adjusted life years (Q ALYs) to value healthcare services.

9. Gafni A, Birch S: Preferences for outcomes in economic evaluation: an economic approach to addressing economic problems. Soc. Sci. M ed. 40(6), 767-776 (1995).
- Provides an overview of some of the general criticisms of Q ALYs and their use.

10. Tsuchiya A, D olan P: The Q ALY model and individual preferences for health states and health profiles over time: a systematic review of the literature. $M$ ed. D ecis. $M$ aking 25 , 460-467 (2005)

- G ood systematic review of the literature that addresses two key assumptions that must hold for QALYs to be used in decision making.

11. Payne K, N icholls S, M cAllister M et al.: O utcome measures for clinical genetics services: a comparison of genetics healthcare professionals and patients' views. $\mathrm{H}$ ealth Policy $84,112-122$ (2007).

12. Fargher EA, Eddy $\mathrm{C}, \mathrm{N}$ ewman $\mathrm{W}$ et al.: Patients' and healthcare professionals' views on pharmacogenetic testing and its future delivery in the N H S. Pharmacogenomics 8(11), 1511-1519 (2007). 
- Presents patients and healthcare professionals' views on the services for pharmacogenetics that must be developed to ensure testing is useful in clinical decision making.

13. Elliott RA, Payne K: Essentials of Economic Evaluation in $\mathrm{H}$ ealth Care. The Pharmaceutical Press, London, UK (2005).

14. Smith R: Construction of the contingent valuation market in health care: a critical assessment. H ealth Economics 12, 609-628 (2003).

- Explains the key issues to consider when designing a contingent valuation study.

15. Phillips KA, Van Bebber SL: A systematic review of cost-effectiveness analysis of pharmacogenomic interventions. Pharmacogenomics 5(8), 1139-1149 (2004).

-. G ood systematic review of published cost- effectiveness analysis of pharmacogenetic technologies.

16. Rogowski W: Current impact of gene technology on healthcare. A map of economic assessments. H ealth Policy 80(2), 340-357 (2007).
17. Marra CA, Esdaile JM, Anis AH: Practical pharmacogenetics: the cost effectiveness of screening for thiopurine S-methyltransferase polymorphisms in patients with rheumatological conditions treated with azathioprine. J. Rheumatol. 29(12), 2507-2512 (2002)

18. Winter J, Walker A, Shapiro D, Gaffney D, Spooner RJ, M ills PR: Cost-effectiveness of thiopurine methyltransferase genotype screening in patients about to commence azathioprine therapy for treatment of inflammatory bowel disease. Aliment. Pharmacol. Ther. 20(6), 593-599 (2004)

19. Oh KT, Anis AH, Bae SC:

Pharmacoeconomic analysis of thiopurine methyltransferase polymorphism screening by polymerase chain reaction for treatment with azathioprine in Korea. Rheumatology 43(2), 156-163 (2004).

20. Dubinsky M C, Reyes E, O fman J, Chiou CF, Wade S, Sandborn WJ: A cost-effectiveness analysis of alternative disease management strategies in patients with Crohn's disease treated with azathioprine or 6-mercaptopurine. Am. J. Gastro. 100(10), 2239-2247 (2005).
21. Priest VL, Begg EJ, Gardiner SJ et al.: Pharmacoeconomic analyses of azathioprine, methotrexate and prospective pharmacogenetic testing for the management of inflammatory bowel disease. Pharmacoeconomics 24(8), 767-781 (2006).

22. van den Akker-van M arle M E, Gurwitz D, D etmar SB et al.: C ost-effectiveness of pharmacogenomics in clinical practice: a case study of thiopurine methyltransferase genotyping in acute lymphoblastic leukemia in Europe. Pharmacogenomics 7(5), 783- 792 (2006).

23. Phillips $K A$, Veenstra $D$, Van Bebber $S L$, Sakowski J: An introduction to cost-effectiveness and cost-benefit analysis of pharmacogenomics. Pharmacogenomics 4(3), 231-239 (2003).

- Provides a useful introduction to the methods of economic evaluation, with a specific focus on pharmacogenetic technologies.

24. Flowers $C R$, Veenstra $D: T$ he role of cost-effectiveness analysis in the era of pharmacogenomics. Pharmacoecnomics 22(8), 481-493 (2004).

Website

101. D epartment of Clinical Biochemistry, City H ospital, Birmingham, UK www.cityassays. org.uk/tpmt.html 